\title{
Evaluating Permeability Surface-Area Product as a Measure of Blood-Brain Barrier Permeability in a Murine Model
}

\author{
(D)E.K. Weidman, (DC.P. Foley, (D) O. Kallas, (D).P. Dyke, (D)A. Gupta, (D)A.E. Giambrone, (D). Ivanidze, (D) H. Baradaran,
} (DD.J. Ballon, and IDP.C. Sanelli

\begin{abstract}
BACKGROUND AND PURPOSE: Permeability surface-area product has been suggested as a marker for BBB permeability with potential applications in clinical care and research. However, few studies have demonstrated its correlation with actual quantitative measurements of BBB permeability. Our aim was to demonstrate the correlation of quantitative permeability surface-area product and BBB permeability in a murine model by histologic confirmation.
\end{abstract}

MATERIALS AND METHODS: Coronal MR imaging was performed on mice treated with mannitol $(n=6)$ for disruption of the BBB and controls treated with saline $(n=5)$. Permeability surface-area product was determined by ROI placement and was compared between saline- and mannitol-treated mice. Correlation was made with contrast-enhancement measurements and immunohistologic-stained sections of tripeptidyl peptidase-1 distribution in mice treated with mannitol and saline followed by injection of a viral vector containing the CLN2 gene, which directs production of tripeptidyl peptidase-1.

RESULTS: Significantly increased permeability surface-area product was seen in mannitol- compared with saline-treated mice in the whole brain $(P=.008)$, MCA territory $(P=.014)$, and mixed vascular territories $(P=.008)$. These findings were compared with contrastenhancement measurements of BBB permeability and were correlated with immunohistologic-stained sections demonstrating BBB permeability to a large vector.

CONCLUSIONS: Permeability surface-area product is increased in situations with known disruptions of the BBB, as evidenced by immunologic staining of large-vector passage through the BBB and concordance with contrast-enhancement measurements in a murine model. Quantitative permeability surface-area product has potential as an imaging marker of BBB permeability.

ABBREVIATIONS: $\mathrm{ACA}=$ anterior cerebral artery; $\mathrm{BBBP}=$ blood-brain barrier permeability; $\mathrm{PCA}=$ posterior cerebral artery; $\mathrm{PS}=$ permeability surface-area product

$D$ eveloping a method of measuring BBB permeability is important for investigating the role of BBB disruption in the pathogenesis of many neurovascular diseases. The BBB is a highly regulated interface between the cerebral intravascular space and the brain parenchyma and comprises endothelial cells, astrocyte end-feet, the capillary basement membrane, and pericytes. In

Received August 19, 2015; accepted after revision December 7.

From the Department of Radiology (E.K.W., C.P.F., O.K., J.P.D., A.G., J.I., H.B., D.J.B., P.C.S.), New York-Presbyterian Hospital, Weill Cornell Medical College, New York, New York; Division of Biostatistics and Epidemiology Department of Healthcare Policy and Research (A.E.G.), Weill Cornell Medical College, New York, New York; and Department of Radiology (P.C.S.), Northwell Health, Manhasset, New York.

D.J. Ballon and P.C. Sanelli are senior authors who contributed equally to this work.

This work was supported by grant 5K23NS058387-02 from the National Institute of Neurological Disorders and Stroke, a component of the National Institutes of Health, to Pina C. Sanelli.

Paper previously presented in its preliminary form at: Eastern Neurological Society Annual Meeting, July 31 to August 3, 2014; Manchester Village, Vermont, and at American Society of Neuroradiology Annual Meeting, April 27, 2015; Chicago, Illinois.

normal conditions, tight junctions between cerebral endothelial cells form a highly selective diffusion barrier that prevents most intravascular solutes from entering the brain. ${ }^{1}$ In some neurovascular diseases, these tight junctions are compromised; this compromise leads to increased BBB permeability and a cascade of pathologic events. For example, in stroke, there is increased BBB permeability that contributes to the development of cerebral edema and hemorrhagic transformation following ischemia. ${ }^{2,3}$ Following hemorrhagic stroke, increased BBB permeability due to thrombin activation ${ }^{4}$ and endothelial cell retraction ${ }^{5}$ results in vasogenic edema. In ischemic stroke, lack of adenosine triphosphate and instigation of a neuroinflammatory response with activation of matrix metalloproteinase- $9^{6,7}$ and changes

Please address correspondence to Pina C. Sanelli, MD, MPH, FACR, Department of Radiology, Northwell Health, 300 Community Dr, Manhasset, NY 11030; e-mail: psanelli@nshs.edu

- Indicates open access to non-subscribers at www.ajnr.org

http://dx.doi.org/10.3174/ajnr.A4712 
in aquaporin-4 expression ${ }^{8}$ contribute to a breakdown of homeostatic BBB functions. Increased pressure along the impaired $\mathrm{BBB}$ following reperfusion contributes to vasogenic

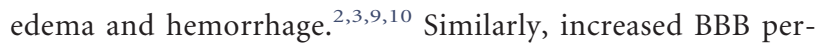
meability following subarachnoid hemorrhage has also been associated with cerebral edema, delayed cerebral ischemia, and poor clinical outcomes. ${ }^{11-13}$

Recently, there have been new advances in imaging BBB permeability. CT perfusion and dynamic contrast-enhanced MR perfusion imaging have been used to evaluate the integrity of the BBB and have been proposed as guides for clinical decision-making in patients with stroke and subarachnoid hemorrhage. Mathematic algorithms based on dynamic contrast enhancement of the brain have been developed to noninvasively quantify BBB permeability. Permeability surface-area product (PS) has been suggested as a promising imaging marker for BBB permeability and measures contrast agent flow through blood vessel walls per unit volume of brain while accounting for the blood flow rate in the vessel and the hematocrit level. PS measurements may better reflect physiologic changes in the BBB compared with traditional contrast-enhancement measurements in the tissue, which measure volume of extracted contrast in the extravascular extracellular space instead of the flow rate across the vessel wall, and thus PS may represent a more accurate assessment of permeability. In the evaluation of the reliability of PS, animal models can provide in vivo imaging and histologic correlates of permeability. While several animal models have been developed to measure BBB permeability, many of these models use extravasation of Evans blue dye, radiolabeled markers, or quantitative fluorescence as permeability measures. ${ }^{14-19}$ Few models have been developed to test noninvasive quantitative imaging of BBB permeability, such as PS, that can be used in human clinical research and clinical practice.

Here, we propose an experimental murine model for quantitative BBB permeability imaging. The purpose of this study was to correlate quantitative PS with gadolinium-enhancement measurements and histologic confirmation of large-vector passage through the BBB in a murine model with known increase in BBB permeability.

\section{MATERIALS AND METHODS \\ Animal Preparation and Immunohistologic Staining}

Experiments were performed on adult male CD-1 mice (Charles River Laboratories, Wilmington, Massachusetts) weighing 22-24 g as previously published. ${ }^{20,21}$ All animal experiments were approved by our Institutional Animal Care and Use Committee. Mice were divided into 2 groups: mice used for immunohistologic staining and mice used for MR imaging gadolinium-enhancement measurements. Both groups underwent identical preparation with mannitol or saline injection in the same murine model. In brief, both groups underwent a standard procedure for anesthesia induced via isoflurane inhalation. The right external carotid artery was dissected and ligated, and a polyimide endovascular microcatheter was introduced into the lumen of the external carotid artery via small arteriotomy. The catheter was rotated so that the tip was oriented in the right ICA distal to the carotid bifurcation. Mice studied for immunohistochemical analysis of BBB permeability received, intra-arterially, $750 \mu \mathrm{L}$ of $25 \%$ weight/volume mannitol solution, a hyperosmolar agent known to disrupt the $\mathrm{BBB},{ }^{22}$ followed after 1 minute by administration of an adeno-associated viral vector containing the CLN2 gene, known to direct the production of the lysosomal enzyme tripeptidyl peptidase-1, produced as previously published. ${ }^{23}$ Tripeptidyl peptidase- 1 distribution in the mouse brain, which has been used as a surrogate marker of BBB permeability, was assessed 5 weeks after gene transfer by immunoperoxidase staining of sagittal sections of the brain. Mice studied for MR perfusion analysis had right ICA catheters attached to a syringe mounted on an MR imaging-compatible programmable syringe pump.

\section{MR Imaging Protocol, Postprocessing, and Data Collection}

Mice were imaged on a 7T small-animal MR imaging system (BioSpec 70/30 USR; Bruker BioSpin, Billerica, Massachusetts). We examined treatment groups with mannitol injection $(750 \mu \mathrm{L}$ of $25 \%$ weight/volume mannitol, $n=6$ ) and the control group with saline injection $(750 \mu \mathrm{L}, n=5)$ at a constant flow rate of 1000 $\mu \mathrm{L} / \mathrm{min}$. Two minutes after either mannitol or saline injection, the mice were injected intra-arterially with $750 \mu \mathrm{L}$ of Gd-DTPA (1:19 dilution in saline of Magnevist; Bayer HealthCare Pharmaceuticals, Wayne, New Jersey) at a rate of $800 \mu \mathrm{L} / \mathrm{min}$ while we monitored the distributed volume and tissue uptake/washout of the injected agent in real-time by using a FLASH gradient-echo pulse sequence with $\mathrm{TR}=25 \mathrm{~ms}, \mathrm{TE}=3.8 \mathrm{~ms}$, FOV $=25 \times 25$ $\mathrm{mm}$, and matrix size $=192 \times 256$. One midbrain coronal imaging frame was captured every 3.6 seconds before, during, and after the Gd-DTPA injection.

Postprocessing of the acquired images into permeability surface-area product (milliliter milliliter ${ }^{-1}$ minute $^{-1}$, volume of liquid per volume brain per minute) and contrast enhancement were performed by using Olea Sphere 2.2 and 2.3 (Olea Medical, La Ciotat, France). PS is described by the permeability model of St. Lawrence and Lee ${ }^{24}$ and characterizes contrast agent flow through blood vessel walls per unit volume of brain, providing a quantitative measure of BBB permeability. PS is described by the relationship ${ }^{24,25}$ accounting for the blood flow rate in the vessel $(F)$ and hematocrit level $(H c t)$ :

$$
\mathrm{PS}=-F(1-H c t) \times \log (1-E),
$$

where $F$ represents cerebral blood flow per unit volume of tissue, corrected for the presence of blood cells with hematocrit $(H c t)$, estimated at 0.45 with average mouse hematocrit reported at $39 \%-49 \%,{ }^{26}$ and $E$ represents the extraction constant, the fraction of contrast that leaves the intravascular space on the first pass of bolus through the vasculature. PS increases with elevated BBB permeability, representing increased flow of contrast through the leaky BBB. The postprocessing technique was standardized for all subjects with the arterial input function placed at the center of the right ICA in a similar fashion for all mice, with representative arterial enhancement time curves shown in Fig 1.

Contrast enhancement was measured as the area under the tissue density curve and peak enhancement over baseline (peak 


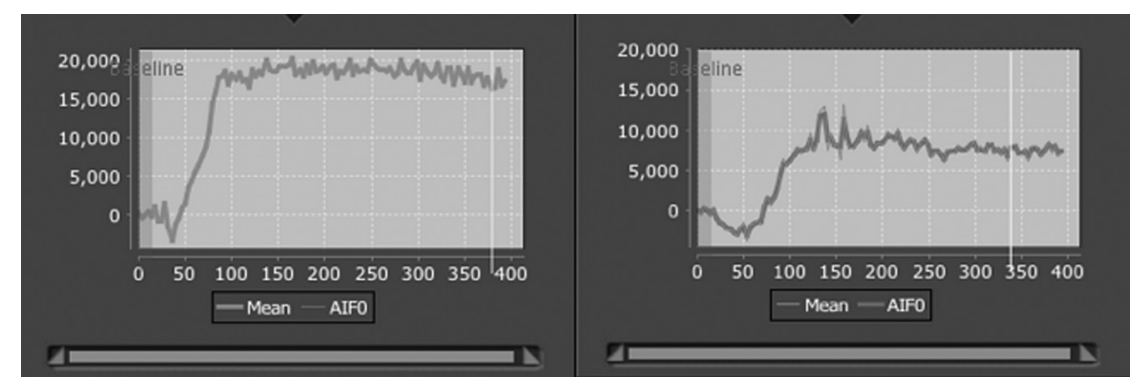

FIG 1. Arterial enhancement time curves in mannitol-treated (left) and saline control (right) mice.
The 2 ROIs in the ACA territory were excluded from ratio-based statistical analysis due to the azygous supply of the ACA territory, with expected resultant contamination of mannitol to the contralateral ACA territory. ${ }^{27}$ Mean ROI ratios for each vascular territory (MCA, PCA, and mixed thalamic/hypothalamic supply) were calculated as well as the mean ROI ratio across all territories for each mouse, generating 1 parameter value per mouse, and were compared between high-dose mannitol and saline-treated groups by using the nonparametric Wilcoxon rank sum test.

Quantitative gadolinium-enhancement data were analyzed by calculating area under the tissue density curve and peak enhancement over baseline for each ROI. Area under the tissue density curve and peak enhancement were compared with mean PS values for each ROI and PS ratio values (excluding the ACA territory) by using the Spearman rank correlation. Immunohistologic staining was quantified as previously described. ${ }^{21}$ Briefly, color images were converted to gray-scale binary images, and an ROI was drawn around the cerebrum. The relative staining efficiency was determined by calculating the number of stained voxels relative to total number of voxels within the ROI. Staining efficiency for mice treated with mannitol and those treated with saline was compared by using the nonparametric Wilcoxon test. All statistical analyses were performed by a biostatistician by using SAS, Version 9.3 (SAS Institute, Cary, North Carolina).

FIG 2. ROI placement on a coronal section of the mouse. ROls 1-12 are in the injected hemisphere with mirror ROIs 13-24 in the contralateral noninjected hemisphere. ACA (1-2, 13-14), MCA (3-6, 15-18), PCA $(7-8,19-20)$, and mixed vascular territories $(9-12,21-24)$ are represented in the image.

enhancement), defined as $\left(S_{1}-S_{0}\right) / S_{0}$, where $S_{1}$ is the signal intensity at the time of maximum enhancement and $S_{0}$ is the signal intensity at baseline. Contrast-enhancement curves for each vascular territory were generated from representative ROI placement.

Quantitative analysis was conducted by using a standardized method with contiguous ROI placement, measuring $1.0 \mathrm{~mm}^{2}$, sampling the cerebral cortex, thalamus, and hypothalamus. Each injected hemisphere had 12 ROIs distributed in the following regions: 2 ROIs in the medial cortex (corresponding to anterior cerebral artery [ACA] territory), 4 ROIs in the lateral cortex (MCA territory), 2 in the hippocampus (posterior cerebral artery [PCA]), and 4 in the thalamic/hypothalamic territory (mixed vascular supply), ${ }^{27-29}$ with mirror ROIs placed in the contralateral control hemisphere (Fig 2).

\section{Statistical Analysis}

Quantitative imaging data were analyzed by calculating the mean PS for each ROI for each mouse. Parameter values of zero were excluded from analysis because this exclusion was consistent with noise artifacts. The ratio of the mean PS value of each ROI in the injected hemisphere to the mean PS value of the corresponding ROI in the contralateral-mirrored (noninjected) hemisphere was calculated to further control for noise variance in each ROI value.

\section{RESULTS}

Twenty-four ROIs were placed on the coronal images of 6 mannitol-treated mice and 5 saline control mice. Only 2 ROIs (ROI 6, MCA territory; and ROI 10, mixed vascular territory) generated PS values of zero in a saline-treated mouse, consistent with generated noise in the image, and were not included in the analysis. No ROIs were excluded in the mannitol-treated mice. When we analyzed the noninjected-hemisphere ROIs (ROIs 13-24), there was no statistically significant difference between mannitoltreated and saline-treated mice in the mean PS $(P=.89)$ or PS by vascular territory (ACA, $P=.32$; MCA, $P=.54$; PCA, $P=.38$; mixed vascular territory, $P=.18$ ) in these hemispheres, confirming that the noninjected sides for both comparison groups were quantitatively similar as would be expected because no intervention occurred in these hemispheres.

Analysis of the injection-side ROIs showed that PS was higher in mannitol-treated mice than in saline-treated mice in all comparisons (Table).

When we compared mean ratios across all ROIs, PS was significantly increased in the mannitol-treated mice $(n=6)$ versus saline control mice $(n=5)(P=.008)$. Relative staining efficiency was calculated from immunohistologic-stained slides of mannitol-treated $(n=3)$ and saline-treated $(n=3)$ mice. Statistically significant increased relative staining efficiency was seen in mannitol-treated mice (median, 3.73; interquartile range, 2.15-7.53) versus saline-treated mice (median, 1.13; interquartile range, 0.53-2.73) $(P=.030)$.

AJNR Am J Neuroradiol 37:1267-74 Jul 2016 www.ajnr.org 
Comparison of mean ROI ratio (treated hemisphere/contralateral noninjected hemisphere) by hemisphere and vascular territory between mannitol- and saline-treated mice

\begin{tabular}{lccc}
\hline Comparison & Mannitol $(\boldsymbol{n}=\mathbf{6})$ & Saline $(\boldsymbol{n}=\mathbf{5})$ & $\boldsymbol{P}$ Value \\
\hline PS ratio & & & \\
Total & $6.35(3.67)$ & $1.32(0.59)$ & .008 \\
MCA & $3.50(1.75)$ & $0.99(0.60)$ & .014 \\
PCA & $8.39(9.23)$ & $3.42(1.91)$ & .315 \\
Mixed & $8.18(6.05)$ & $0.53(0.30)$ & .008 \\
\hline
\end{tabular}

${ }^{a}$ Data are presented as mean (SD).

When we compared vascular territories, a statistically significant increased PS was seen in mannitol-treated versus salinetreated mice in the MCA $(P=.014)$ and mixed vascular territories $(P=.008)$. We observed a trend toward increased PS in mannitoltreated mice in the PCA territory $(P=.315)$ (Fig 3$)$. Due to differences in sectioning, qualitative comparison with the histologic correlate by vascular territories was performed, demonstrating increased vector staining in high-dose mannitol-treated mice in similar vascular territories (Fig 3). Particularly high areas of vector passage and staining were seen in the mixed vascular territory (hypothalamus), MCA territory (striatum and medial cerebral cortex), and PCA territory (superior colliculus).

Contrast-enhancement data and curves were generated for comparison with PS findings (Fig 4). Contrast-enhancement curves and quantitative data represent the contrast extravasation into the extravascular extracellular tissue of the brain. The highest peaks of contrast-enhancement curves were seen in the PCA territory in both mannitol-treated and saline control mice and corresponded to the high absolute PS values measured in these regions. A statistically significant moderate correlation was found between absolute PS with area under the tissue density curve and peak enhancement over baseline separately $(R=0.61, R=0.58$, $P<.0001$ ) (Fig 5), as well as between the PS ratio with area under the tissue density curve and peak enhancement over baseline $(R=$ $0.50, R=0.59, P<.0001)$.

\section{DISCUSSION}

In this study, we have described an experimental mouse model for measuring $\mathrm{BBB}$ permeability by using PS derived from MR imaging data. We demonstrated increased PS, a quantitative surrogate imaging measure of $\mathrm{BBB}$ permeability that could easily be adapted in clinical care, in the mouse brain with known disruption of the BBB. When we used imaging acquired after the same method of $\mathrm{BBB}$ disruption (route, dose, and rate of mannitol delivery) described for large-vector BBB passage, PS was statistically increased in the whole injected hemisphere and particularly in the MCA and mixed vascular territories compared with the control group. These findings correlated with contrast-enhancement measurements and corresponded to the increased transgene expression secondary to large-vector passage in the whole brain as measured by relative staining efficiency. Quantitative measures of bloodbrain barrier permeability (BBBP) by using PS offer less interreader variability than qualitative assessment alone and have the potential for use in both clinical care and research to evaluate regional and global changes in BBBP with time.

This is the first study, to our knowledge, to evaluate the use of quantitative PS derived from MR imaging data in a murine model with histologically demonstrated increased BBB permeability. Previous studies have reported correlations between other dy- namic contrast-enhanced MR imaging permeability parameters and histologic or auto-radiographic measures of permeability in animal models. For example, Hoffmann et $\mathrm{al}^{17}$ demonstrated a correlation between increased permeability measures by using the Patlak model and areas of histologically identified Evans blue extravasation and hemorrhage in a rat ischemic stroke model following MCA occlusion. The contrast transfer coefficient has been reported to correlate with increased BBB permeability as measured by auto-radiography in a rat brain tumor model. ${ }^{30}$ Similarly, our study found increased PS with known BBB disruption, as confirmed by immunohistologic staining. In contrast to models using Evans blue, which measures the volume of dye in the extracellular space, our study parameter PS measured the flow and the leakage (volume) across the BBB. Most important, our study evaluated an imaging marker of BBBP with the potential for use in clinical care and research in humans because it does not require tissue staining and postmortem analyses.

Our sample size allowed us to detect statistically significant differences in quantitative PS between mannitol- and salinetreated mice in the whole injected hemisphere and in the MCA and mixed vascular territories. In addition, our data showed a trend toward increased PS in the PCA territory of mannitol- versus saline-treated mice. With increased sample size, we may also be able to detect a statistically significant difference between the mannitol and saline groups in the PCA territory, which represented the smallest vascular territory included in the analysis, comprising only 2 ROIs per hemisphere. The PCA territory demonstrated the highest PS values in both saline- and mannitoltreated mice, thus requiring a larger sample size to adequately power this subanalysis in our study. Increased enhancement and increased transgene expression were seen in the PCA-supplied superior colliculus and hippocampus on the histologic sections in mannitol-treated mice, corresponding to the high PS values seen in the PCA territory. The finding of increased PS in the PCA territory relative to other territories in both the mannitol- and saline-treated mice may reflect increased vulnerability of the BBB in the PCA territory due to high-pressure injection of saline. This explanation is in keeping with the concept that there is increased susceptibility of cerebrovascular auto-regulatory dysfunction in the PCA territory, as seen in hypertensive encephalopathy with development of interstitial edema. ${ }^{31,32}$

For this initial study, we analyzed single coronal images that were obtained following BBB disruption in mannitol- or salinetreated mice. Histologic sections of a separate group of mice treated with mannitol or saline were made in the sagittal plane, and we compared coronal imaging findings with sagittal histologic samples. Both groups of mice (mice analyzed by MR imaging and mice analyzed by histologic analysis) were the same murine model comprising 22- to 24-g genetically engineered male CD-1 adult mice from the same laboratory receiving equal amounts of mannitol or saline via an identical intra-arterial injection method, representing replicate mice, so we believe that the comparison between groups is valid. To account for the differences in ana- 
MCA Territory PS Ratio
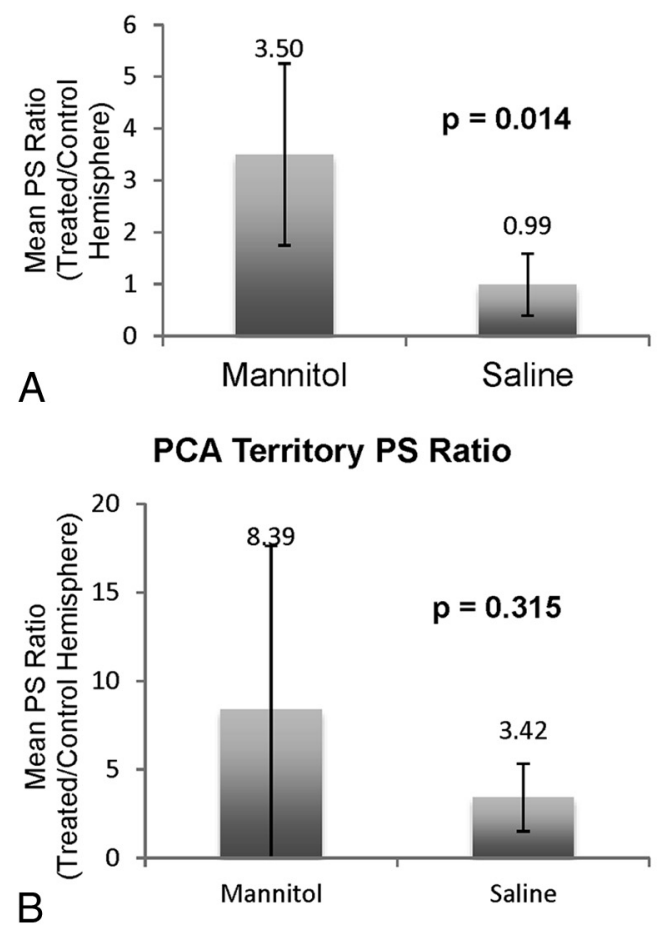

D

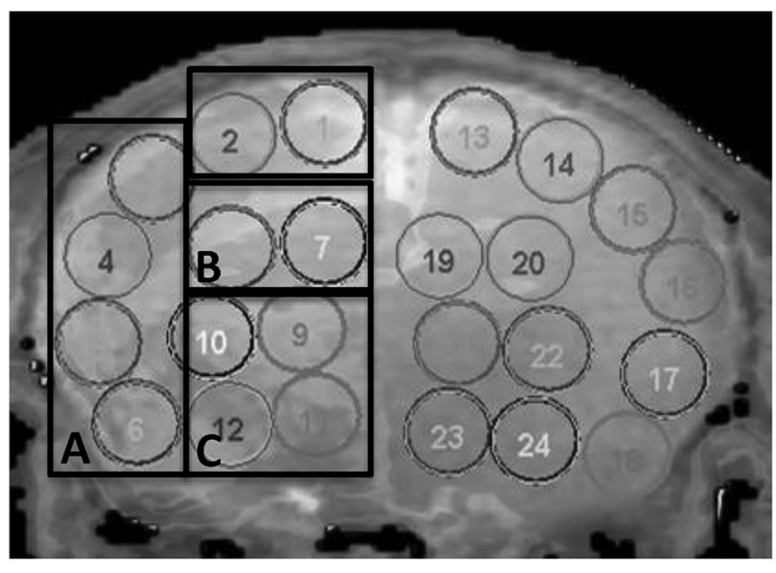

\section{Mixed Vascular Territory PS Ratio}

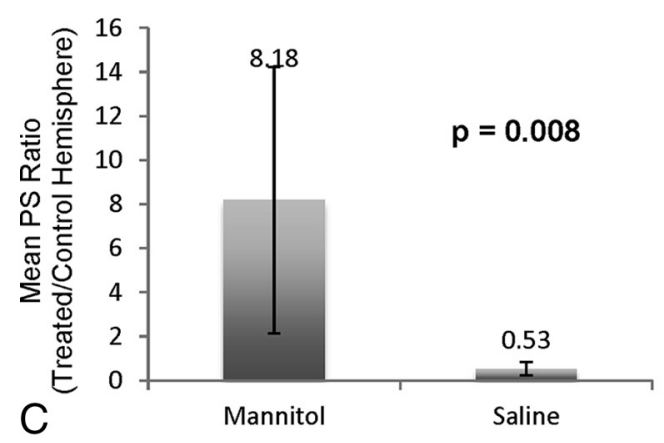

\section{Mannitol treated mouse - Injected hemisphere}

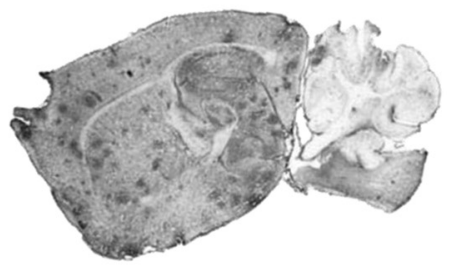

Mannitol treated mouse Non-injected hemisphere

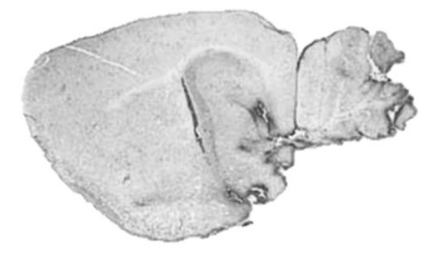

$\mathrm{E}$

\section{Saline treated mouse - Injected hemisphere}

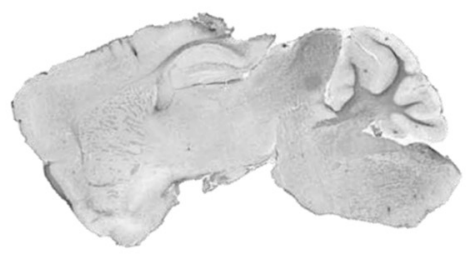

FIG 3. A-C, Mean PS ratio (injected hemisphere/contralateral noninjected hemisphere) per vascular territory in mannitol-treated mice versus saline controls. Top of the column represents the mean value, and error bars represent the SD. D, ROI placement on a representative coronal image. Boxed areas $(A-C)$ correspond to vascular territory ROIs for MCA, PCA, and mixed territories, respectively. E, Immunoperoxidase staining on a sagittal section of a mannitol-treated mouse in the injected hemisphere, its contralateral noninjected hemisphere, and a saline-injected hemisphere demonstrate the effect of mannitol-mediated BBB disruption on transgene expression. Darker regions correspond to increased tripeptidyl peptidase-1 expression.

tomic planes, we qualitatively compared individual vascular territory and whole-hemispheric brain. We quantitatively measured increased staining efficiency in mice treated with mannitol compared with saline-treated mice, confirming that mannitol opened the blood-brain barrier. We expect that BBB permeability after ICA mannitol injection may vary by vascular territory due to collateral circulation; thus, we believe that this is a valid correlation. However, we acknowledge that the study is somewhat limited by the inability to directly quantitatively compare PS and staining efficiency in the same mouse. It may be further valuable for future studies to compare exact planes and both PS and histologic findings in the same subject in correlating imaging with histologic findings.

In this experimental model, direct intra-arterial injection of mannitol and vector into the right ICA has the potential compli- cation of focal or global cerebral ischemia. To control for the possible effect of ischemia/infarction on vector passage and tripeptidyl peptidase-1 expression, we compared mannitol-treated mice with mice that were injected in an identical fashion (same rate and total volume) with saline followed by a vector. While the ICA injection pressure that could potentially lead to ischemia/ infarction or BBBP alterations in these mice is unknown, we believe the saline-treated mice were a valid control for this injection technique. Additionally, no evidence of ischemia or infarction was seen on MR images of the injected mice.

Our model of hyperosmotic mannitol disruption of the $\mathrm{BBB}$ resulted in global BBBP alteration in this study. Mannitol-induced disruption of the BBBP has been reported in other animal models, which demonstrate increased permeability to Evans blue, methotrexate, and the radiolabeled markers carbon 14 sucrose 


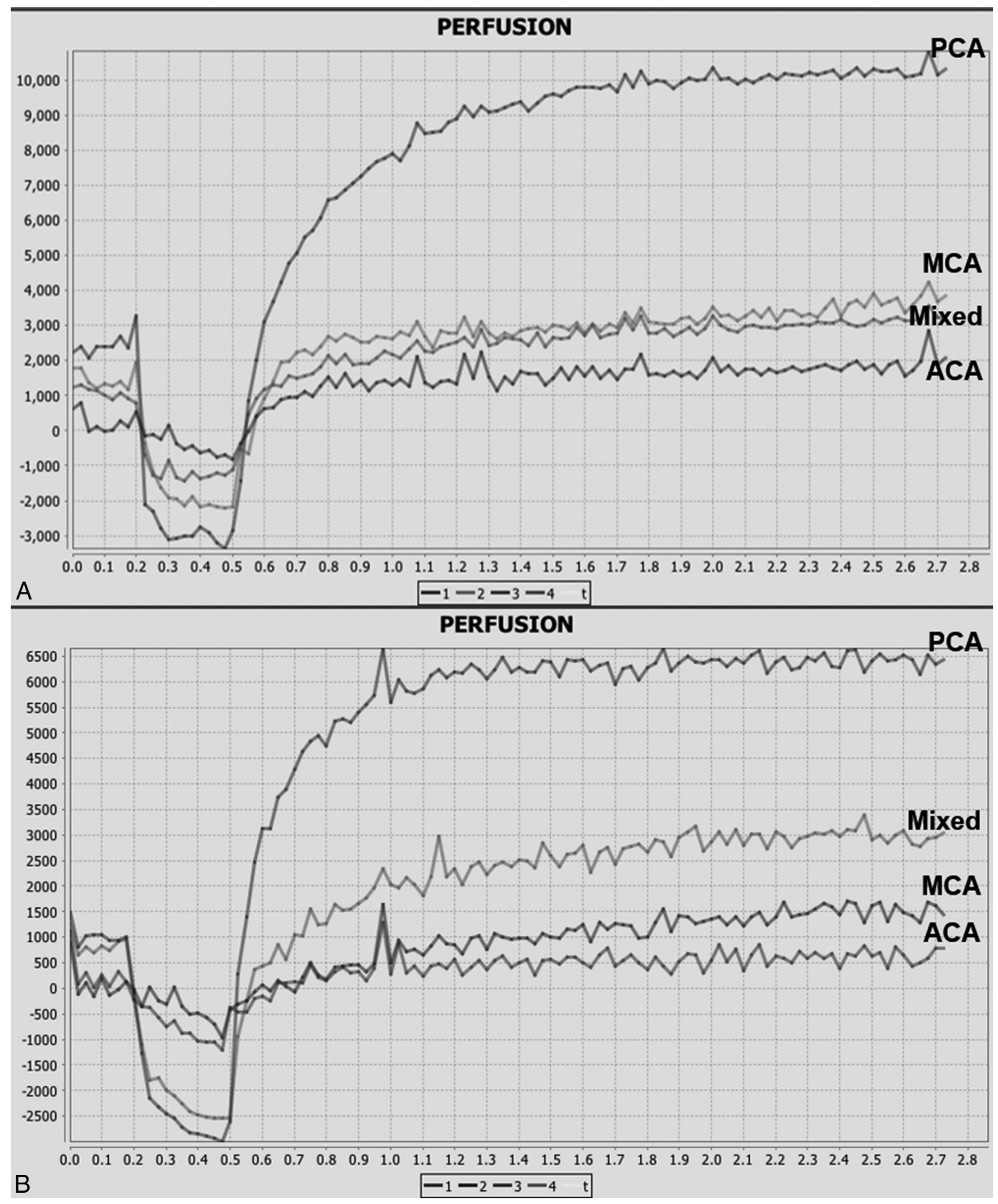

FIG 4. A, Representative contrast-enhancement curves in ROIs corresponding to ACA, MCA, PCA, and mixed vascular territories in a mannitoltreated mouse. $B$, Representative contrast-enhancement curves in ROls corresponding to ACA, MCA, PCA, and mixed vascular territories in a saline-treated control mouse.

and rubidium $86+{ }^{19,33}$ and in clinical use for therapeutic delivery in humans. ${ }^{34,35}$ One study by Cosolo et al $^{33}$ described methotrexate levels 4-5 times higher following intra-arterial mannitol injection compared with the noninjected hemisphere in rats, in keeping with our total-brain PS comparison between mannitol- and saline-treated mice. Even though this murine model of global mannitol-induced disruption of the BBB does not directly study specific neurovascular diseases with regional alterations of permeability as seen in stroke and cerebral ischemia, we believe that these findings would be applicable in situations with both 


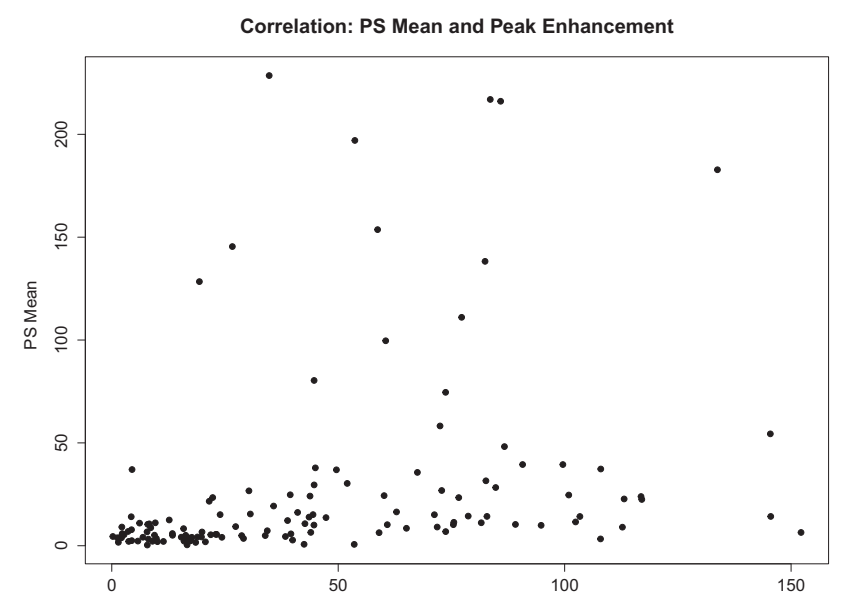

A

Peak Enhancement
Spearman's $r=0.58, p=<.0001$

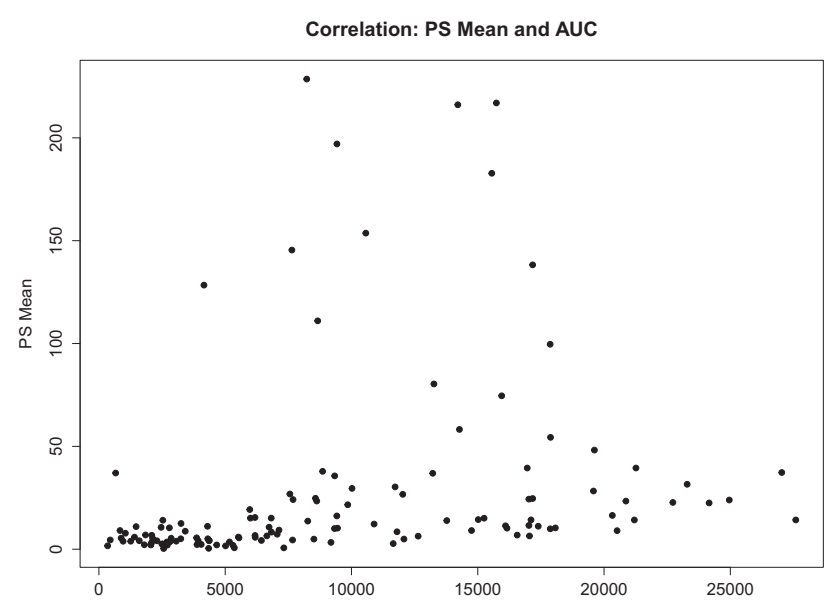

B

Spearman's $\mathrm{AUC}$ =0.61, $\mathrm{p}=<.0001$

FIG 5. Scatterplots of PS versus contrast-enhancement parameters $A, P S$ mean versus peak enhancement. $B$, PS mean versus area under the tissue density curve.

global and regional increased BBBP because we are studying the correlation of increased PS with increased barrier permeability as measured by contrast enhancement and histologic staining, rather than studying the specific underlying pathophysiologic mechanisms leading to the BBB disruption. Additionally, global changes in BBBP have been reported following subarachnoid hemorrhage ${ }^{11,12}$ with global cerebral edema and posttraumatic brain injury. While the aim of this initial proofof-concept study has been to evaluate PS as a measure of BBBP, our study additionally adds to the literature further evidence of the use of mannitol for global BBBP disruption, which may be helpful in assessing pathologic conditions resulting in global cerebral edema.

We have demonstrated that PS correlates with disruption of the BBB by using Gd-DTPA and histologic staining. These results are accurate for the molecular weight of Gd-DTPA used in this study. We have not assessed other contrast agents of different molecular weights (eg, gadolinium-labeled albumin) in our analyses. Further evaluation of PS, which depends on the extraction constant and cerebral blood flow and hematocrit, with different molecular weights of gadolinium is warranted.

\section{CONCLUSIONS}

We have demonstrated the use of quantitative PS derived from MR imaging data as a surrogate imaging measure of BBB permeability in a murine model with gadolinium enhancement and histologic confirmation of increased barrier permeability. This study demonstrates the use of perfusion imaging to produce a quantitative measure of $\mathrm{BBB}$ permeability confirmed by histologic analysis, which could be translatable to other animal models of disease. Most important, these findings support the implementation of quantitative PS as a surrogate imaging marker of BBB permeability in future clinical research and, potentially, decision-making in clinical care.

\section{ACKNOWLEDGMENTS}

The authors thank Gulce Askin for assistance with this study.
Disclosures: Pina C. Sanelli_RELATED: Grant: National Institute of Neurological Disorders and Stroke (5K23NS058387-02). Conor P. Foley-RELATED: Grant: National Institutes of Health-National Institute of Neurological Disorders and Stroke, Comments: Ruth L. Kirchstein F32 postdoctoral fellowship, F32 NS073397/NS/NINDS NIH HHS/United States; UNRELATED: Patents (planned, pending or issued): Cornell University, Comments: patent for neural drug-delivery technology; Royalties: Cornell University, Comments: royalties on a patent for neural drug-delivery technology. Jana Ivanidze-UNRELATED: Grants/ Grants Pending: Radiological Society of North American Resident Research Grant 2014-2015.

\section{REFERENCES}

1. Ballabh P, Braun A, Nedergaard M. The blood-brain barrier: an overview: structure, regulation, and clinical implications. Neurobiol Dis 2004;16:1-13 CrossRef Medline

2. Lin K, Kazmi KS, Law M, et al. Measuring elevated microvascular permeability and predicting hemorrhagic transformation in acute ischemic stroke using first-pass dynamic perfusion CT imaging. AJNR Am J Neuroradiol 2007;28:1292-98 CrossRef Medline

3. Hom J, Dankbaar JW, Soares BP, et al. Blood-brain barrier permeability assessed by perfusion CT predicts symptomatic hemorrhagic transformation and malignant edema in acute ischemic stroke. AJNR Am J Neuroradiol 2011;32:41-48 CrossRef Medline

4. Xi G, Hua Y, Bhasin RR, et al. Mechanisms of edema formation after intracerebral hemorrhage: effects of extravasated red blood cells on blood flow and blood-brain barrier integrity. Stroke 2001;32: 2932-38 CrossRef Medline

5. Satpathy M, Gallagher P, Lizotte-Waniewski M, et al. Thrombininduced phosphorylation of the regulatory light chain of myosin II in cultured bovine corneal endothelial cells. Exp Eye Res 2004;79: 477-86 CrossRef Medline

6. Rosenberg GA, Estrada EY, Dencoff JE. Matrix metalloproteinases and TIMPs are associated with blood-brain barrier opening after reperfusion in rat brain. Stroke 1998;29:2189-95 CrossRef Medline

7. Asahi M, Wang X, Mori T, et al. Effects of matrix metalloproteinase-9 gene knock-out on the proteolysis of blood-brain barrier and white matter components after cerebral ischemia. $J$ Neurosci 2001;21:7724-32 Medline

8. Ribeiro Mde C, Hirt L, Bogousslavsky J, et al. Time course of aquaporin expression after transient focal cerebral ischemia in mice. J Neurosci Res 2006;83:1231-40 CrossRef Medline

9. Unterberg AW, Stover J, Kress B, et al. Edema and brain trauma. Neuroscience 2004;129:1021-29 Medline

10. Strbian D, Durukan A, Pitkonen M, et al. The blood-brain barrier is 
continuously open for several weeks following transient focal cerebral ischemia. Neuroscience 2008;153:175-81 CrossRef Medline

11. Doczi T. The pathogenetic and prognostic significance of bloodbrain barrier damage at the acute stage of aneurysmal subarachnoid haemorrhage: clinical and experimental studies. Acta Neurochir (Wien) 1985;77:110-32 CrossRef Medline

12. Germanò A, d'Avella D, Imperatore C, et al. Time-course of bloodbrain barrier permeability changes after experimental subarachnoid haemorrhage. Acta Neurochir (Wien) 2000;142:575-80; discussion 580-81 CrossRef Medline

13. Sercombe R, Dinh YR, Gomis P. Cerebrovascular inflammation following subarachnoid hemorrhage. Jpn J Pharmacol 2002;88:227-49 CrossRef Medline

14. Gotoh $\mathrm{O}$, Asano $\mathrm{T}$, Koide $\mathrm{T}$, et al. Ischemic brain edema following occlusion of the middle cerebral artery in the rat, I: the time courses of the brain water, sodium and potassium contents and bloodbrain barrier permeability to $125 \mathrm{I}$-albumin. Stroke 1985;16:101-09 CrossRef Medline

15. Belayev L, Busto R, Zhao W, et al. Quantitative evaluation of bloodbrain barrier permeability following middle cerebral artery occlusion in rats. Brain Res 1996;739:88-96 CrossRef Medline

16. Uyama O, Okamura N, Yanase M, et al. Quantitative evaluation of vascular permeability in the gerbil brain after transient ischemia using Evans blue fluorescence. J Cereb Blood Flow Metab 1988;8: 282-84 CrossRef Medline

17. Hoffmann A, Bredno J, Wendland MF, et al. Validation of in vivo magnetic resonance imaging blood-brain barrier permeability measurements by comparison with gold standard histology. Stroke 2011;42:2054-60 CrossRef Medline

18. Hatashita S, HoffJT. Brain edema and cerebrovascular permeability during cerebral ischemia in rats. Stroke 1990;21:582-88 CrossRef Medline

19. Brown RC, Egleton RD, Davis TP. Mannitol opening of the bloodbrain barrier: regional variation in the permeability of sucrose, but not $86 \mathrm{Rb}+$ or albumin. Brain Res 2004;1014:221-27 CrossRef Medline

20. Santillan A, Rubin DG, Foley CP, et al. Cannulation of the internal carotid artery in mice: a novel technique for intra-arterial delivery of therapeutics. J Neurosci Methods 2014;222:106-10 CrossRef Medline

21. Foley CP, Rubin DG, Santillan A, et al. Intra-arterial delivery of AAV vectors to the mouse brain after mannitol mediated blood brain barrier disruption. J Control Release 2014;196:71-78 CrossRef Medline

22. Zünkeler B, Carson RE, Olson J, et al. Quantification and pharma- cokinetics of blood-brain barrier disruption in humans. J Neurosurg 1996;85:1056-65 CrossRef Medline

23. Sondhi D, Hackett NR, Peterson DA, et al. Enhanced survival of the LINCL mouse following CLN2 gene transfer using the rh.10 rhesus macaque-derived adeno-associated virus vector. Mol Ther 2007;15: 481-91 CrossRef Medline

24. St Lawrence KS, Lee TY. An adiabatic approximation to the tissue homogeneity model for water exchange in the brain, I: theoretical derivation. J Cereb Blood Flow Metab 1998;18:1365-77 Medline

25. Permeability Models: A Review [manual]. La Ciotat, France: Olea Medical

26. Reference Values for Laboratory Animals: Normal Hematologic Values. Research Animal Resources, University of Minnesota. http:// www.ahc.umn.edu/rar/refvalues.html. Accessed February 25, 2015

27. Dorr A, Sled JG, Kabani N. Three-dimensional cerebral vasculature of the CBA mouse brain: a magnetic resonance imaging and micro computed tomography study. Neuroimage 2007;35:1409-23 CrossRef Medline

28. Watson C, Paxinos G, Puelles L, eds. The Mouse Nervous System. Amsterdam: Elsevier Academic Press; 2012:xvii

29. Lein ES, Hawrylycz MJ, Ao N, et al. Genome-wide atlas of gene expression in the adult mouse brain. Nature 2007;445:168-76 CrossRef Medline

30. Ferrier MC, Sarin H, Fung SH, et al. Validation of dynamic contrastenhanced magnetic resonance imaging-derived vascular permeability measurements using quantitative autoradiography in the RG2 rat brain tumor model. Neoplasia 2007;9:546-55 CrossRef Medline

31. Sadoshima S, Fujii K, Yao H, et al. Regional cerebral blood flow autoregulation in normotensive and spontaneously hypertensive rats: effects of sympathetic denervation. Stroke 1986;17:981-84 CrossRef Medline

32. Engelter ST, Provenzale JM, Petrella JR. Assessment of vasogenic edema in eclampsia using diffusion imaging. Neuroradiology 2000; 42:818-20 CrossRef Medline

33. Cosolo WC, Martinello P, Louis WJ, et al. Blood-brain barrier disruption using mannitol: time course and electron microscopy studies. Am J Physiol 1989;256:R443-47 Medline

34. Rapoport SI. Osmotic opening of the blood-brain barrier: principles, mechanism, and therapeutic applications. Cell Mol Neurobiol 2000;20:217-30 CrossRef Medline

35. Rodriguez A, Tatter SB, Debinski W. Neurosurgical techniques for disruption of the blood-brain barrier for glioblastoma treatment. Pharmaceutics 2015;7:175-87 CrossRef Medline 